\title{
Sífilis congénita con hídrops fetal: reporte de cuatro casos en un hospital general de referencia en Bogotá, Colombia entre 2016- 2020
}

\section{Congenital syphilis with hydrops fetalis: report of four cases in a general referrall hospital in Bogota, Colombia} between 2016-2020

\section{Andrés Mauricio Camacho-Montaño $M D^{1}$; Reinaldo Niño-Alba $M^{1}{ }^{1}$; Edgar Páez- Castellanos $^{1}$}

Recibido: 18 de agosto de 2020/Aceptado: 19 de abril de 2021

\section{RESUMEN}

Objetivo: realizar un reporte de 4 casos de hídrops fetal secundario a sífilis congénita y hacer una revisión de la literatura para responder la siguiente pregunta: ¿cuál es el esquema antibiótico utilizado en los casos de sífilis gestacional complicada con hídrops fetal?

Materiales y métodos: se presentan 4 casos de sífilis congénita con hídrops fetal. La edad materna varió entre 17 y 28 años, la edad gestacional al momento del diagnóstico estuvo entre 25 y 30 semanas, dos de ellas no habían iniciado control prenatal en ese momento. En tres casos se dio tratamiento para sífilis gestacional inmediatamente con penicilina cristalina entre 6 y 12 semanas antes del parto y se formuló tratamiento a la pareja con penicilina benzatínica. Respecto a los recién nacidos, dos de ellos no tenían infección activa o secuelas, se consideró que uno de ellos tenía sífilis congénita por títulos de prueba no treponémica. En uno de los casos, la pa-

* Correspondencia: Andrés Mauricio Camacho Montaño. Calle $100 \mathrm{n} 48 \mathrm{f}$ 17. Camacho.andresm@gmail.com

1. Subred integrada de servicios de salud centro oriente. E.S.E. Bogotá (Colombia) ciente no alcanza a recibir tratamiento para la sífilis gestacional antes del parto, este recién nacido tenía signos de infección activa. Se hizo una revisión de la literatura en las bases de datos Medline, LILACS y google scholar; los términos de búsqueda fueron los siguientes: "hídrops fetal", "lues", "syphilis - prenatal diagnosis- ultrasound - penicilina - treatment". Se buscaron reportes y series de casos o cohortes de recién nacidos con sífilis gestacional con hídrops fetalis. Se extrajo información sobre la madre y el recién nacido respecto al tratamiento.

Resultados: se identificaron 119 artículos, de estos 13 cumplieron con los criterios de inclusión, tres fueron descartados por no tener acceso al texto completo. Se incluyeron diez estudios de un total de 16 casos reportados con diagnóstico prenatal de hídrops fetal secundarios a infección congénita. De ellos, tres presentaron anemia fetal severa y requirieron transfusión intrauterina; 5 casos recibieron tratamiento intrauterino con penicilina. En cuatro casos la madre recibió penicilina benzatínica intramuscular por 3 semanas, uno recibió además penicilina cristalina endovenosa por 13 días, otro recibió penicilina cristalina endovenosa por 14 días. Un total de 11 casos no recibieron tratamiento 
durante la gestación; 6 de los 16 casos (37,5\%) presentaron muerte perinatal.

Conclusión: el retraso en acudir al control prenatal y la tardanza del diagnóstico y tratamiento de la sífilis gestacional son causas importantes de la persistencia de la sífilis congénita. Se requieren estudios aleatorizados para determinar el mejor tratamiento del feto con sífilis congénita en los 30 días previos al parto y del feto con compromiso sistémico durante la segunda mitad de la gestación.

Palabras clave: hidropesía fetal, sífilis, sífilis congénita

\section{ABSTRACT}

Objective: To report four cases of hydrops fetalis secondary to congenital syphilis and carry out a review of the literature to answer the question, What is the antibiotic regimen used in cases of gestational syphilis with hydrops fetalis as a complication?

Material and methods: Four cases of congenital syphilis with hydrops fetalis are presented. Maternal age ranged between 17 and 28 years, gestational age at the time of diagnosis varied between 25 and 30 weeks, and two of the mothers had not initiated prenatal care at that time. Treatment with crystalline penicillin for gestational syphilis was given immediately 6 to 12 weeks before delivery in three cases and partners were prescribed treatment with benzathine penicillin. As for the neonates, two had no active infection or sequelae and one of them was considered to have congenital syphilis based on non-treponemal test titers. In one case, the patient was unable to receive syphilis treatment before delivery and her newborn had signs of active infection. A review of the literature was conducted in the Medline, LILACS and Google Scholar databases using the search terms "hydrops fetalis," "Lues", "syphilis - prenatal diagnosis ultrasound - penicillin - treatment". The search included case reports and case series or cohorts of newborns with gestational syphilis and hydrops fetalis. Information regarding treatment in the mothers and in the newborns was extracted.
Results: Overall, 119 articles were identified. Of these, 13 met the inclusion criteria, three were discarded because the full text could not be accessed. Ten studies with a total of 16 reported cases of hydrops fetalis secondary to congenital infection were reported. Of these, three presented with severe fetal anemia and required intrauterine transfusion; 5 cases received intrauterine penicillin treatment. In four cases the mother received weekly intramuscular injections of benzathine penicillin for 3 weeks, one received additional intravenous crystalline penicillin for 13 days, while another one received intravenous crystalline penicillin for 14 days. Treatment during gestation was not given in a total of 11 cases; and 6 of the 16 cases (37.5\%) resulted in perinatal death.

Conclusion: Delays in prenatal care and late diagnosis and treatment of gestational syphilis are important causes of persistent congenital syphilis. Randomized studies are required to identify the best treatment in fetuses with congenital syphilis 30 days before delivery and in fetuses with systemic compromise during the second half of gestation.

Keywords: Hydrops fetalis, syphilis, congenital syphilis

\section{INTRODUCCIÓN}

La sífilis es una infección causada por la bacteria treponema pallidum TP, la cual se adquiere principalmente por transmisión sexual (ITS), aunque también por vía sanguínea o a través de la placenta de la madre al feto (1); la infección puede cursar con sintomatología (sífilis primaria, segundaria o terciaria) o de forma asintomática (latente) en el adulto (2); sin embargo, cuando una paciente tiene infección y queda en embarazo o adquiere la infección durante el embarazo se desarrolla la sífilis gestacional (3). La transmisión madre-fetal del TP puede ocurrir en cualquier trimestre (4); Ingraham, citado por Chakraborty, informa que en cualquier estado clínico se puede trasmitir la sífilis al feto, siendo más frecuente en estados tempranos de la enfermedad (70-100\% en sífilis primaria a 40\% en sífilis latente temprana) (3), y aun en gestantes sífilis 
terciaria se han descrito casos de sífilis congénita (5). La infección fetal es principalmente transplacentaria, aunque puede ocurrir durante el parto en presencia de lesiones genitales (3); el compromiso del feto o del recién nacido es usualmente devastador, cuando la sífilis gestacional no es diagnosticada ni tratada oportunamente (1). Clínicamente, la sífilis congénita se puede dividir en temprana (primeros dos años de vida), donde se encuentran, anormalidades óseas, hepatomegalia, esplenomegalia, petequias, lesiones en la piel, entre otras; y tardía (después de dos años) que se acompaña principalmente de múltiples secuelas (queratitis intersticial, distrofias dentales, deformidad nasal, prominencia frontal, deformación palatina, entre otras) $(3,5)$.

En el 2012 la Organización Mundial de la Salud (OMS) estimó 930,000 casos de sífilis gestacional en el mundo, que causaron cerca de 350,000 resultados adversos del embarazo y 200,000 casos de abortos o mortinatos (6); en los últimos años en Estados Unidos se ha visto un repunte de casos, los Centros de Control de Enfermedades (CDC) reportaron una incidencia creciente de sífilis congénita, de 8,4 por 100,000 Nacidos Vivos (NV) para 2012 a 33,1 por 100,000 NV en 2018, siendo la población hispana una de las de mayor riesgo (44,7 por 100,000 NV) (7). Las cifras en Colombia también muestran un aumento de la incidencia de sífilis congénita de 0,6 casos por 1,000 NV en 2016 a 1,2 casos por 1,000 NV en 2020 que es concordante con el aumento de la razón de prevalencia de sífilis gestacional en los mismos años que pasó de 3,6 por 1,000 NV a 6,5 por 1,000 NV (8). Algunos factores propuestos que podrían contribuir a esta elevación de casos en el mundo son la falta de acceso a la atención prenatal temprana, el uso de sustancias psicoactivas, el incremento de la migración, el bajo cumplimiento por parte de los proveedores de salud de las pautas nacionales, la escasa financiación para las ITS como problema de salud pública, las múltiples parejas sexuales, inicio temprano de las relaciones sexuales, ITS concomitantes, entre otras (9-11). Sin embargo, en cerca de la mitad de los casos no hay un factor de riesgo identificable (12).
El diagnóstico de la sífilis gestacional y congénita se encuentra claramente descrito en guías de manejo de las sífilis gestacional y congénita internacionales (1, 2) y nacionales (13). El método más común para evaluar la infección fetal es la ecografía de detalle donde se identifican los estigmas de la transmisión in útero (14), estos se pueden identificar consistentemente después de las 20 semanas cuando el feto es inmunocompetente para generar la respuesta inflamatoria robusta necesaria para producir anormalidades e incluyen hepatomegalia (79\%), placentomegalia (27\%), polhidramnios (12\%), ascitis (10\%) y alteraciones en la velocidad sistólica pico de la arteria cerebral media (MCA PSV por sus iniciales en inglés) en el Doppler y otros signos de hídrops fetal (33\%); aunque en la mayoría de los casos no se reporta ningún hallazgo $(15,16)$. No está clara la fisiopatología del hídrops fetal en la infección por sífilis; se cree que la destrucción hepática directa (principal órgano hematopoyético fetal) por el treponema lleva a anemia fetal severa que se suma a la sepsis fetal con daño endotelial e incremento de la permeabilidad capilar, resulta finalmente en falla cardiaca junto con fuga de plasma a través de las uniones endoteliales, llevando al estado hidrópico (16).

El tratamiento de la sífilis gestacional está basado en el tiempo de la infección, en sífilis temprana $(<1$ año de duración) se sugiere Penicilina benzatínica G 2.4 millones de unidades intramusculares (IM) una sola dosis; la sífilis tardía ( $>1$ año de duración), de duración desconocida o terciaria (enfermedad cardiovascular o lesiones gomatosas) se trata con Penicilina benzatínica G 2.4 millones de unidades intramusculares (IM) semanales por tres semanas $(13,17)$.

Aunque la evidencia de efectividad y seguridad del tratamiento antibiótico de los recién nacidos con sífilis congénita (18) es considerada de baja certeza, derivada principalmente de estudios observacionales, estos resultados apoyan el uso de penicilina para el tratamiento de los recién nacidos afectados por sífilis congénita, con penicilina cristalina 100 000-150 $000 \mathrm{U} / \mathrm{kg} /$ día por vía intravenosa de 10 
a 15 días, o penicilina procaínica $50000 \mathrm{U} / \mathrm{kg} /$ día dosis única por vía intramuscular de 10 a 15 días, como con recomendación fuerte a favor, en algunas de las guías disponibles $(13,17)$. El tratamiento de la neurosífilis o sífilis ocular se trata con Penicilina G cristalina 3-4 millones de unidades intravenosas (IV), cada 4 horas entre 10 y 14 días (2). La comparación entre esquemas basados en penicilina benzatínica y procaínica no parecieran mostrar diferencias en términos de manifestaciones clínicas de sífilis congénita o cura serológica (18). Por otra parte, hay discrepancia en el tratamiento de recién nacidos con sífilis congénita menos probable, la guía del Centers for Diseases Control and Prevention (CDC) en Estados Unidos y la guía de práctica clínica basada en la evidencia para la atención integral de la sífilis gestacional y congénita en Colombia, recomiendan el uso de Penicilina benzatínica G 50.000 unidades IM en dosis única $(2,13)$; mientras que la guía del New Zealand Sexual Health Society (NZSHS) recomienda seguimiento a los recién nacidos sin tratamiento específico y solo el uso de la dosis de 50.000 unidades en casos de difícil seguimiento (19). Por otra parte, aunque es claro que el tratamiento oportuno de la sífilis gestacional es suficiente para prevenir la sífilis congénita, si se administra 30 días antes del parto $(20,21)$, no es claro cuál es el mejor tratamiento de la sífilis en el feto cuando la infección se detecta en los últimos 30 días antes del parto, a su vez, es poca la información publicada sobre el manejo del feto con sífilis congénita e hídrops fetal, ya que se ha descrito que se requeriría tratamiento de la madre con penicilina cristalina (22). Por lo tanto, el objetivo de este estudio es reportar cuatro casos de embarazos complicados por hídrops fetal secundario a sífilis congénita, así como hacer una revisión de la literatura del tratamiento del recién nacido con hídrops fetal y su resultado perinatal.

\section{REPORTE DE CASOS}

Los casos que se describirán a continuación se recolectaron entre el 2017 y 2019 en el hospital La Victoria, institución pública hospitalaria de referencia, ubicada en la ciudad de Bogotá, atiende principalmente a población del régimen subsidiado por el Estado en el sistema de salud en Colombia. La tabla 1 muestra un resumen de los principales hallazgos.

\begin{tabular}{|c|c|c|c|c|c|c|c|}
\hline \multicolumn{8}{|c|}{ Resumen de los casos. } \\
\hline Caso & $\begin{array}{c}\text { Edad } \\
\text { materna }\end{array}$ & $\begin{array}{c}\text { EG dx } \\
\text { hídrops }\end{array}$ & $\begin{array}{l}\text { EG dx } \\
\text { Sífilis }\end{array}$ & Tratamiento & EG nacer & Peso al nacer & $\begin{array}{c}\text { Resultado } \\
\text { perinatal }\end{array}$ \\
\hline Caso 1 & 17 años & $25.5 \mathrm{~s}$ & $25,5 \mathrm{~s}$ & $\begin{array}{c}\text { Penicilina IV } \\
14 \text { días a la } \\
\text { madre. }\end{array}$ & $37,0 \mathrm{sem}$ & $2820 \mathrm{gr}$ & $\begin{array}{l}\text { Sobrevida } \\
\text { neonatal. }\end{array}$ \\
\hline Caso 2 & 28 años & $28.4 \mathrm{~s}$ & $28,4 \mathrm{~s}$ & $\begin{array}{l}\text { Penicilina IV } \\
14 \text { días a la } \\
\text { madre y también } \\
\text { al recién nacido. }\end{array}$ & $37,0 \mathrm{sem}$ & $2325 \mathrm{gr}$ & $\begin{array}{l}\text { Sobrevida } \\
\text { neonatal. }\end{array}$ \\
\hline Caso 3 & 20 años & $30.2 \mathrm{~s}$ & $16.0 \mathrm{~s}$ & $\begin{array}{l}\text { Penicilina ben- } \\
\text { zatínica IM } 3 \\
\text { dosis a la madre. } \\
\text { Tratamiento al } \\
\text { recién nacido } \\
\text { para SC. }\end{array}$ & $30.2 \mathrm{sem}$ & $1260 \mathrm{gr}$ & $\begin{array}{l}\text { Sobrevida } \\
\text { neonatal. }\end{array}$ \\
\hline Caso 4 & 18 años & $29.0 \mathrm{~s}$ & $29.0 \mathrm{~s}$ & $\begin{array}{l}\text { Penicilina IV } 14 \\
\text { días a la madre. }\end{array}$ & $36,2 \mathrm{sem}$ & $2460 \mathrm{gr}$ & $\begin{array}{l}\text { Sobrevida } \\
\text { neonatal. }\end{array}$ \\
\hline
\end{tabular}

$\mathrm{IV}=$ Intravenosa 


\section{CASO 1}

Mujer de 17 años, oriunda y residente de Bogotá, en unión libre, que ingresa a la institución el 03 de agosto de 2017. Fue remitida del servicio de consulta externa al servicio de urgencias, con gestación de 25,5 semanas, por hallazgos ecográficos anormales. Antecedentes médicos negativos. Antecedentes gineco-obstétricos: 2 gestaciones, 1 parto, sin abortos previos. Inició el control prenatal en la semana 11, y realizó tres controles prenatales sin alteraciones. Se realizaron dos pruebas treponémicas rápidas negativas.

Examen físico: frecuencia cardiaca 78 latidos por minuto (lpm), presión arterial 105/ 60 mmHg. Altu- ra uterina de $25 \mathrm{~cm}$ y fetocardia $149 \mathrm{lpm}$. Hallazgos positivos: altura uterina de $26 \mathrm{~cm}$ y fetocardia 139 latidos por minuto (LPM). Se toman paraclínicos: hemograma normal: $\mathrm{Hb}: 11 \mathrm{mg} / \mathrm{dl}$, creatinina: 0.5 mg/ dl. Hepatitis B: negativo, prueba VIH: negativa, prueba treponémica rápida: positiva, prueba no treponémica (VDRL) positiva en 1: 64 diluciones. Se realiza el diagnóstico de caso probable de sífilis gestacional. Ecografía: biometría para 25 semanas, crecimiento fetal normal. Hallazgos positivos: hídrops fetal con presencia de ascitis, derrame pericárdico, hidrocele bilateral; además, presencia de hepatomegalia y oligoamnios. ILA. $4 \mathrm{~cm}$. El doppler evidencia anemia fetal severa (Figura 1).

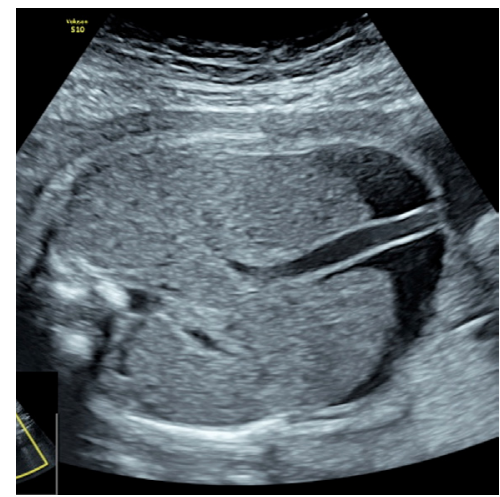

a. Ascitis.

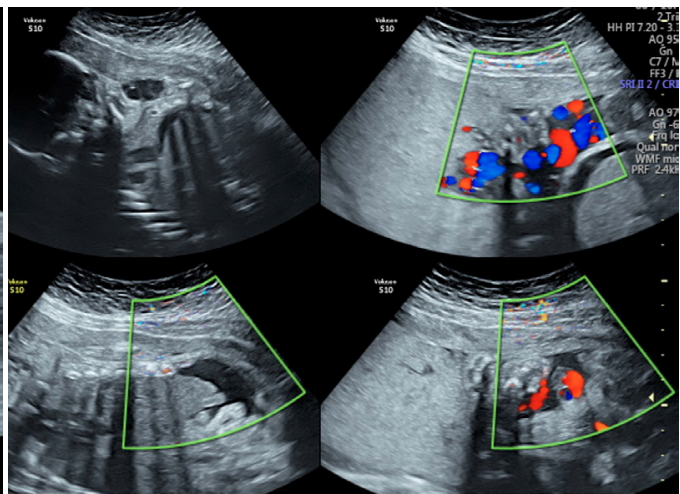

b. Oligomanios.

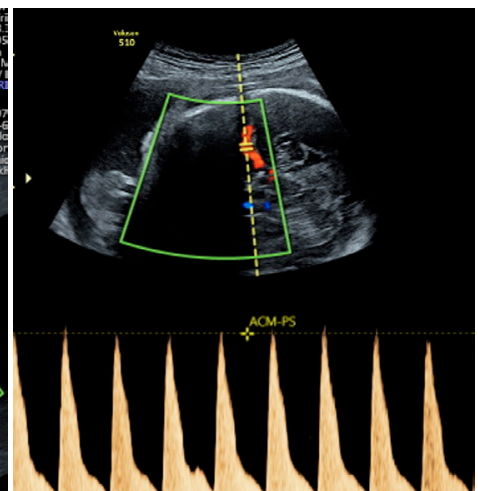

C. Doppler arteria cerebral media.

Figura 1. Ultrasonido de feto con infección sifilítica a las 25 semanas e hídrops fetalis. Fuente: elaboración propia.

Se hospitalizó, se inició esquema de maduración pulmonar fetal con betametasona, neuroprotección fetal con sulfato de magnesio y tratamiento con penicilina endovenosa de 5 millones de unidades cada 4 horas por 14 días por considerarse un caso de sífilis congénita con afectación fetal severa. Se realizó tratamiento a la pareja de la paciente con penicilina benzatínica. Evolución: se llevó a cabo control ultrasonográfico a las 24 horas del ingreso. Doppler de arteria cerebral media, anemia leve. Paciente con evolución clínica satisfactoria. Control a los 7 días, doppler de arteria cerebral media normal. Líquido amniótico normal. Persistencia de ascitis y hepatomegalia. Control a los 14 días. Doppler de anemia cerebral media normal. Líquido amniótico normal. Persistencia hepatomegalia y mejoría de hídrops fetal sin derrame pericárdico y disminución de la ascitis. Se dio salida y control ecográfico semanal. En la semana 37 inició trabajo de parto espontáneo. Prueba treponémica rápida positiva. VDRL 2 diluciones. Conducción del parto sin complicaciones, se atiende parto vaginal obteniendo producto de sexo masculino, de peso 2,820 gramos, con adaptación neonatal adecuada. A las 24 horas del puerperio se da salida de la madre sin complicaciones. El recién nacido fue hospitalizado para estudio, recibió profilaxis con penicilina benzatínica de $50.000 \mathrm{UI} / \mathrm{Kg} / \mathrm{IM}$ dosis única, estudio de sífilis congénita negativo (VDRL no reactiva, hemograma, radiografía de huesos largos, líquido cefalorraquídeo, uroanálisis y función hepática) por lo que se dio salida. 


\section{CASO 2}

Mujer de 28 años que ingresó a la institución el 18 de julio de 2017, estado civil soltera, oriunda de Quibdó y residente en Bogotá. Consultó al servicio de urgencias por dolor pélvico, presentaba gestación de 28,4 semanas. Antecedentes médicos negativos. Antecedentes gineco-obstetricos: 2 gestaciones, 1 parto, ninguna cesárea ni abortos. Sin controles prenatales.

Examen físico: frecuencia cardiaca 100 lpm, presión arterial 120/ $70 \mathrm{mmHg}$. Altura uterina de $28 \mathrm{~cm}$ y fetocardia $144 \mathrm{lpm}$. Se toman paraclínicos. Hemograma: leucocitos 10300 x mcl, neutrófilos 70\% Hb:11 gr/dl, hematocrito 33, plaquetas 326 000 x mcl, proteína $C$ reactiva $20 \mathrm{mg} / \mathrm{L}$, creatinina: $0,7 \mathrm{mg} / \mathrm{dl}$, hepatitis B: negativa, prueba VIH: negativa, prueba treponémica rápida: positiva. VDRL: 1024 diluciones. Se realizó diagnóstico de caso confirmado de sífilis gestacional. Ultrasonido obstétrico mostró: biometría para 26 semanas. Cre- cimiento fetal normal. Líquido amniótico normal. Hallazgos positivos: hídrops fetal con presencia de ascitis, derrame pericárdico e hidrocele bilateral; además, presencia de hepatomegalia (Figura 2). Se hospitalizó, se inició esquema de maduración pulmonar fetal, neuroprotección fetal con sulfato de magnesio y tratamiento con penicilina endovenosa de 5 millones de unidades cada 4 horas por 14 días por considerar sífilis gestacional con afectación fetal severa. Se dio tratamiento a la pareja con penicilina benzatínica 3 dosis. Evolución: el Doppler de arteria cerebral media sin evidencia de anemia fetal, en la hospitalización se realizó monitoria fetal por turno. Control con ecografía y doppler a los 7 días: doppler de anemia cerebral media normal. Líquido amniótico normal. Persistencia de ascitis y hepatomegalia. Control ecográfico a los 14 días: Doppler de anemia cerebral media normal. Líquido amniótico normal, persistencia hepatomegalia, sin ascitis. Se da salida y control ecográfico semanal.

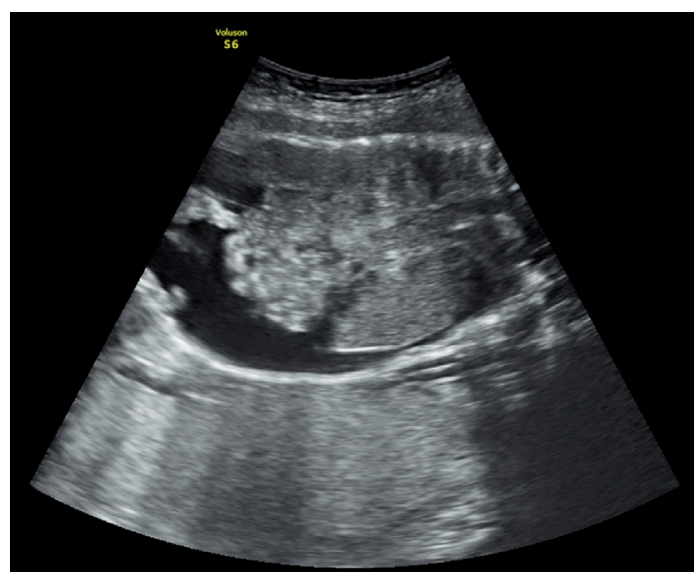

a. Ascitis.

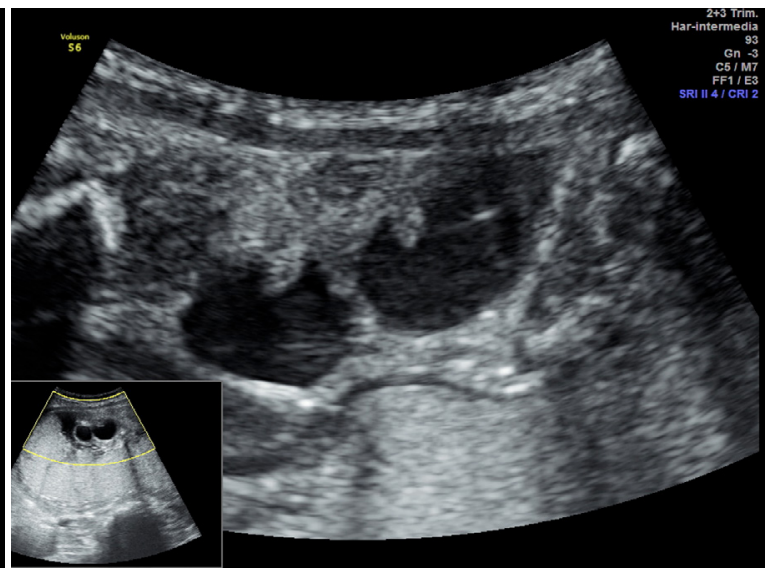

b. Hidrocele.

Figura 2. Ultrasonido de feto con infección sifilítica a las 258 semanas e hídrops fetalis. Fuente: elaboración propia.

En la semana 37 ingresó la paciente en trabajo de parto, fase latente, se realizó refuerzo con oxitocina; durante el trabajo de parto presentó cifras de presión arterial en rango de severidad que cedieron con nifedipino. Se inició sulfato de magnesio para profilaxis de eclampsia. Paraclínicos de perfil toxémico en límites normales. VDRL reportado: 1, 1024 diluciones. Valorada por el servicio de infectología quien formula penicilina benzatínica 2,400,000 UI IM, una dosis semanal por tres semanas; y a la pareja quien refiere no cumplió la indicación inicial de tratamiento. Se atendió parto vaginal obteniendo producto de sexo masculino de peso 2,325 g con adaptación neonatal adecuada. A las 72 horas del puerperio se dio salida de la madre sin complicaciones. Sin antihipertensivo. Al recién nacido se le toma VDRL a las 12 horas: 1:16 diluciones, se rea- 
lizó estudio para sífilis congénita y recibió tratamiento con penicilina endovenosa por 14 días, posterior a lo cual se dio egreso sin complicaciones.

\section{CASO 3}

Mujer de 20 años que ingresó a la institución el 10 de octubre de 2019, estado civil soltera, oriunda y residente de Bogotá. Consultó al servicio de urgencias por actividad uterina con gestación de 30.2 semanas, sin otros síntomas. Antecedentes médicos negativos. Antecedentes gineco-obstétricos: 2 gestaciones, 1 partos, sin abortos previos, un hijo vivo. Paciente inició controles prenatales en semana 16, se documentó sífilis gestacional, se administraron 3 dosis de penicilina $G$ benzatínica de 2,400,000 de unidades intramuscular. Se dio tratamiento a la pareja.

Examen físico: signos vitales en parámetros normales, frecuencia cardiaca 84 lpm, presión arterial 110 /66 $\mathrm{mmHg}$. Frecuencia respiratoria 20, altura uterina de $29 \mathrm{~cm}$ fetocardia $134 \mathrm{lpm}$, al examen ginecológico: dilatación 4 cm con membranas íntegras. Se tomaron paraclínicos: hemograma: $\mathrm{Hb}: 11 \mathrm{gr} / \mathrm{dl}$, creatinina: 0,5 mg/ dl, hepatitis B: negativa, prueba VIH: negativa, prueba treponémica rápida: positiva y VDRL: 64 diluciones. Se realizó el diagnóstico de caso probable de sífilis gestacional. Se considera reinfección y se formula penicilina benzatínica al compañero. Ultrasonido: biometría para 30 semanas. Crecimiento fetal normal. Líquido amniótico normal. Hídrops fetal: derrame pericárdico, ascitis; además de hepatomegalia y ventriculomegalia simétrica leve. Doppler de anemia cerebral media sin anemia (Figura 3). Se hospitalizó, se inició esquema de maduración pulmonar fetal, neuroprotección fetal con sulfato de magnesio, tocolisis con nifedipino. Evolución: el monitoreo fetal presenta desaceleraciones, se consideró estado fetal no satisfactorio y se decide cesárea por lo que no alcanza a iniciar el manejo para sífilis gestacional. Procedimiento sin complicaciones. Líquido amniótico fétido y cavidad hipertérmica. Se obtuvo recién nacido de sexo femenino de 1,260 gramos, Silverman de 6 , requirió intubación endotraqueal y traslado a unidad de cuidados intensivos neonatales. La madre inició tratamiento con antibiótico: clindamicina y gentamicina por sospecha clínica de corioamnionitis, con evolución satisfactoria y se da salida al tercer día de puerperio. El recién nacido se hospitalizó para estudio y recibió tratamiento con penicilina cristalina endovenosa por 14 días, posteriormente se dio egreso sin complicaciones.

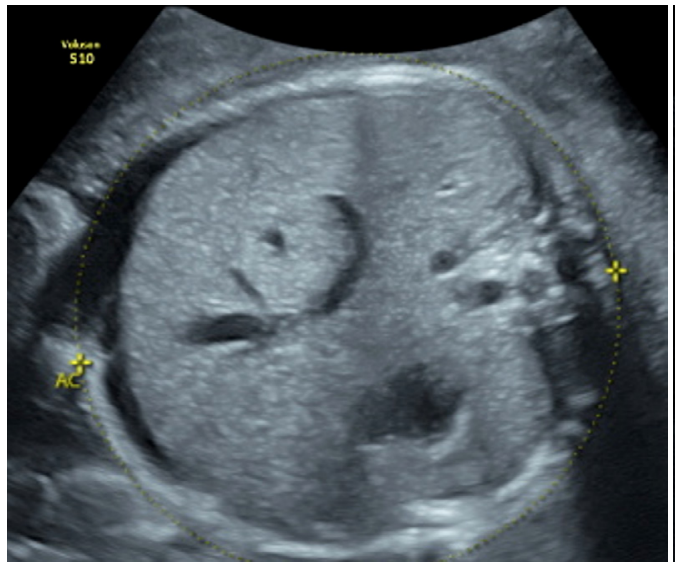

a. Ascitis

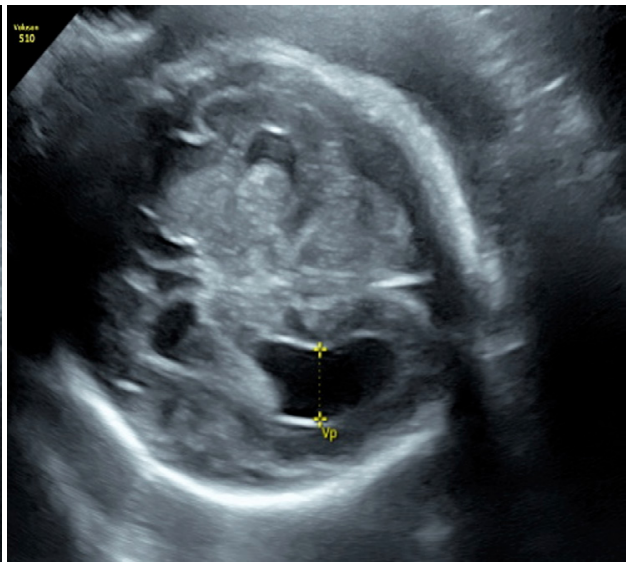

b. Ventriculomegalia.

Figura 3. Ultrasonido de feto con infección sifilítica a las 30 semanas e hídrops fetalis.

Fuente: elaboración propia.

\section{CASO 4}

Mujer de 18 años que ingresó a la institución el 18 de octubre de 2019, estado civil soltera, oriunda de Venezuela y residente en Bogotá desde hace 3 meses. Consultó al servicio de urgencias por dolor pélvico con gestación de 29 semanas. Ninguna otra sintomatología. Antecedentes médicos negativos. Antecedentes gineco-obstétricos: 3 gestaciones, 2 partos, sin abortos, 2 hijos vivos. 
No había iniciado control prenatal hasta ese momento. Examen físico: deprimida con llanto fácil, signos vitales: frecuencia cardiaca 90 lpm, presión arterial 108/70 mmHg. Altura uterina de $28 \mathrm{~cm}$ y fetocardia $144 \mathrm{lpm}$. Hallazgos positivos: altura uterina de $29 \mathrm{~cm}$ fetocardia $150 \mathrm{lpm}$. Se tomaron paraclínicos: hemograma: leucocitos $8450 \mathrm{x}$ mol Hb: 12,5 gr/dl, creatinina: 0,4 mg/ dl, hepatitis B: negativa, prueba VIH: negativa, prueba treponémica rápida: positiva y VDRL positiva en 1: 32 diluciones. Se realizó el diagnóstico de caso probable de sífilis gestacional. Ultrasonido obstétrico: biometría para 30,1 semanas. Crecimiento fetal normal. Líquido amniótico normal. Hallazgos positivos: hídrops fetal con presencia de ascitis, hidrotórax; además, presencia de hepatomegalia. El doppler de arteria cerebral media sin evidencia de anemia fetal (Figura 4). Se

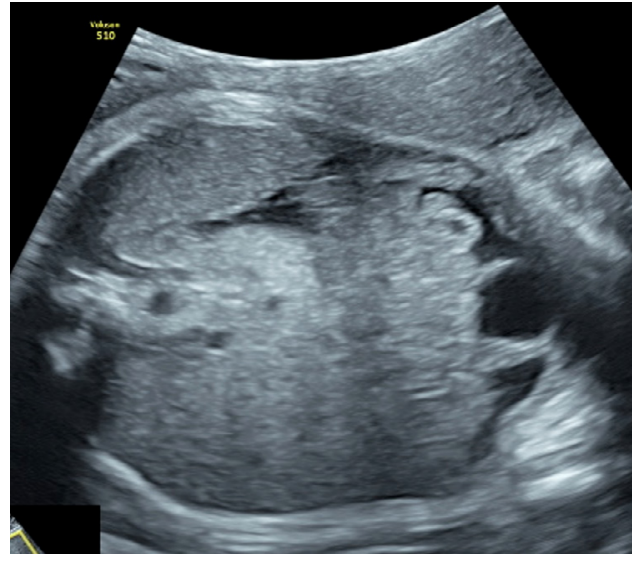

a. Ascitis. decide hospitalizar, esquema de maduración pulmonar fetal, neuroprotección fetal con sulfato de magnesio y tratamiento con penicilina endovenosa de 5 millones de unidades cada 4 horas por 14 días por considerar sífilis gestacional con afectación fetal severa. Se da tratamiento a la pareja con penicilina benzatínica por 3 dosis. Evolución: Se realizó control con ultrasonido a las 24 horas. Doppler de arteria cerebral media: anemia severa. Se realiza control ecográfico a las 48 horas. Doppler de arteria cerebral media: anemia leve y persistencia de hídrops fetal. Paciente con evolución clínica satisfactoria. Control ecográfico a los 7 días. Doppler de anemia cerebral media normal. Líquido amniótico normal. Sin signos de hídrops fetal. Se dio egreso de la institución.

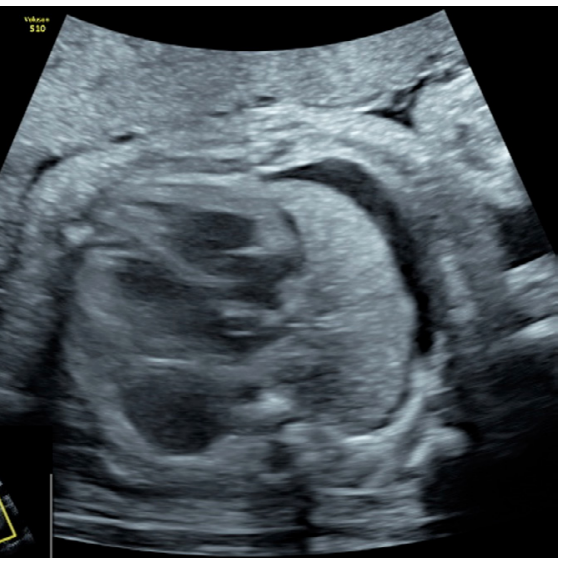

b. Hidrotorax.

Figura 4. Ultrasonido de feto con infección sifilítica a las 29 semanas e hídrops fetalis.

Fuente: elaboración propia.

Inició trabajo de parto espontáneo a las 36 semanas y 2 días. Prueba treponémica rápida: positiva. VDRL negativa, obteniendo recién nacido cuyo peso fue de 2,460 gr, talla $48 \mathrm{~cm}$, perímetro cefálico $32 \mathrm{~cm}$, puntaje APGAR 8-9-9. Se toma control de VDRL a la madre en el ingreso a sala de partos reportado como 1:16 diluciones. El nacimiento sucede sin complicaciones, el examen físico del recién nacido es normal y el VDRL a las 12 horas es de 1:8, se toman paraclínicos para descartar sífilis congénita que son negativos (hemograma, radiografía de huesos largos, líquido cefalorraquídeo, uroanálisis y función hepática). La paciente y el recién nacido permanecen en alojamiento conjunto 24 horas posterior al parto. Se administra penicilina cristalina al recién nacido de manera profiláctica $50.000 \mathrm{UI} / \mathrm{Kg} / \mathrm{IM}$ dosis, y se realiza seguimiento a la semana; no se evidencian complicaciones maternas o del recién nacido.

Consideraciones éticas. Las pacientes fueron informadas del diagnóstico, firmaron consentimiento informado para cada procedimiento realizado y un consentimiento expreso para la publicación de este caso. Se tomaron las precauciones para garantizar la confidencialidad de la información y el anonimato de las pacientes; el registro fotográfico fue tomado por los autores. 


\section{MATERIALES Y MÉTODOS}

Con el objetivo de responder la pregunta ¿cuál es el régimen de penicilina utilizado en el manejo de sífilis gestacional con compromiso fetal severo manifestado por hídrops fetal? Se realizó una revisión sistemática de la literatura con los siguientes términos mesh: hídrops fetal - lues- syphilis - prenatal diagnosis- ultrasound - penicilina - treatment. Se realizó una búsqueda en las siguientes bases de datos: Medline vía pubmed, LILACS OVID, UPTODATE y en Google scholar. La búsqueda se limitó a artículos publicados en inglés y español. La búsqueda tuvo en cuenta estudios de los últimos 30 años. Además, se realizó la técnica "bola de nieve", que tiene por objeto buscar en las referencias de los artículos encontrados. Los tres investigadores realizaron la búsqueda de manera independiente, al final se realizó una conciliación entre todos los autores. Se buscaron reportes y series de casos o cohortes de recién nacidos con sífilis gestacional con hídrops fetalis. Se extrajo información sobre la madre y el recién nacido respecto al diagnóstico y el tratamiento.

\section{RESULTADOS}

Se identificaron 119 títulos, de los cuales 13 estudios cumplieron con los criterios de inclusión, 3 fueron excluidos por no tener acceso al texto completo $(23,24$, 25). Los 10 artículos seleccionados fueron publicados entre 1992 y $2019(26,27,28,29,30,31,32,33,34,35)$ (ver Tabla 2). Siete estudios son reportes de caso $(28,29,30,31,33,34,35)$, y $3(26,27,32)$ son series de casos, que a su vez presentan 9 casos entre los 3 estudios, para un total de 16 casos.

\begin{tabular}{|c|c|c|c|c|c|c|c|c|}
\hline & & Casos rep & ortados c & Hídrops & $\begin{array}{l}\text { bla } 2 \text {. } \\
\text { etal secundar }\end{array}$ & os a sífilis & ongénita. & \\
\hline $\begin{array}{l}\text { Autor- } \\
\text { año }\end{array}$ & País & $\begin{array}{c}\text { Edad } \\
\text { materna }\end{array}$ & $\begin{array}{c}\text { EG dx } \\
\text { hídrops }\end{array}$ & $\begin{array}{l}\text { EG dx } \\
\text { Sífilis }\end{array}$ & Tratamiento & EG nacer & Peso al nacer & $\begin{array}{c}\text { Resultado } \\
\text { perinatal }\end{array}$ \\
\hline \multirow{4}{*}{$\begin{array}{l}\text { Bercowitz } \\
1990(26)\end{array}$} & \multirow{4}{*}{ USA } & 26 & 32 & Postnatal & No & 32 & 2525 & $\begin{array}{l}\text { Sobrevida } \\
\text { neonatal. }\end{array}$ \\
\hline & & 21 & 32 & Postnatal & No & 32 & 1460 & $\begin{array}{l}\text { Sobrevida } \\
\text { neonatal. }\end{array}$ \\
\hline & & 36 & Término & Postnatal & No & término & ND & $\begin{array}{c}\text { Muerte } \\
\text { neonatal } \\
\text { temprana. }\end{array}$ \\
\hline & & 30 & 27 & 27 & No & 27 & 2070 & $\begin{array}{c}\text { Muerte } \\
\text { intrauterina. }\end{array}$ \\
\hline \multirow{3}{*}{$\begin{array}{c}\text { Barton } \\
1992(27)\end{array}$} & \multirow{3}{*}{ USA } & 22 & 31 & 31 & $\begin{array}{l}\text { Penicilina } \\
\text { IM }\end{array}$ & término & 3540 & $\begin{array}{l}\text { Sobrevida } \\
\text { neonatal. }\end{array}$ \\
\hline & & 18 & 35 & 35 & No & 35 & 2700 & $\begin{array}{l}\text { Sobrevida } \\
\text { neonatal. }\end{array}$ \\
\hline & & 21 & 34 & 34 & No & 34 & 2530 & $\begin{array}{l}\text { Sobrevida } \\
\text { neonatal. }\end{array}$ \\
\hline $\begin{array}{c}\text { Galan } \\
1993(28)\end{array}$ & USA & 21 & 24 & 24 & $\begin{array}{c}\text { Penicilina } \\
\text { IM } 3 \text { dosis y } \\
\text { penicilina IV } \\
14 \text { días. }\end{array}$ & 37 & ND & $\begin{array}{l}\text { Sobrevida } \\
\text { neonatal. }\end{array}$ \\
\hline $\begin{array}{c}\text { Levine } \\
1998(29)\end{array}$ & USA & 31 & 29 & Postnatal & No & 29 & 1825 & $\begin{array}{c}\text { Muerte } \\
\text { neonatal } \\
\text { temprana. }\end{array}$ \\
\hline
\end{tabular}




\begin{tabular}{|c|c|c|c|c|c|c|c|c|}
\hline $\begin{array}{c}\text { Chen } \\
2010(30)\end{array}$ & Canadá & 17 & 27 & 28 & $\begin{array}{c}\text { Penicilina IV } \\
14 \text { días. }\end{array}$ & 35 & 2390 & $\begin{array}{l}\text { Sobrevida } \\
\text { neonatal. }\end{array}$ \\
\hline $\begin{array}{c}\text { Araujo } \\
2012(31)\end{array}$ & Brazil & 21 & 25 & 7 & $\begin{array}{c}\text { Penicilina IM } \\
3 \text { dosis }\end{array}$ & 33 & 3095 & $\begin{array}{l}\text { Sobrevida } \\
\text { neonatal. }\end{array}$ \\
\hline \multirow{2}{*}{$\begin{array}{c}\text { Mace } \\
2014(32)\end{array}$} & \multirow{2}{*}{ Francia } & 17 & 34 & Postnatal & No & 35 & 2300 & $\begin{array}{l}\text { Sobrevida } \\
\text { neonatal. }\end{array}$ \\
\hline & & 27 & 26 & 17 & No & 26 & 720 & $\begin{array}{c}\text { Muerte } \\
\text { intrauterina. }\end{array}$ \\
\hline $\begin{array}{c}\text { Fuchs } \\
2016(33)\end{array}$ & Canadá & 19 & 23 & 23 & $\begin{array}{l}\text { Penicilina } \\
\text { IM }\end{array}$ & 23 & 858 & $\begin{array}{c}\text { Muerte } \\
\text { intrauterina. }\end{array}$ \\
\hline $\begin{array}{c}\text { Duby } \\
2019(34)\end{array}$ & Canadá & 28 & 28 & 31 & No & 31 & 1710 & $\begin{array}{l}\text { Sobrevida } \\
\text { neonatal. }\end{array}$ \\
\hline $\begin{array}{c}\text { Ramis } \\
\text { 2019(35) }\end{array}$ & España & 23 & 31 & Postnatal & No & 32 & 2510 & $\begin{array}{c}\text { Muerte } \\
\text { neonatal } \\
\text { temprana. }\end{array}$ \\
\hline
\end{tabular}

$\mathrm{IV}=$ Intravenosa

Sitio de publicación: cuatro estudios se publicaron en Estado Unidos $(26,27,28,29)$, tres en Canadá $(30,33,34)$, uno en Francia (32), uno en Brasil (31), y uno en España (35).

La edad materna de los casos publicados está entre los 17 y 33 años $(29,32)$, con un promedio de 22,5 años, la edad gestacional al momento del diagnóstico de la sífilis está entre 7 y $35(27,31)$ semanas, con un promedio de 20,7 semanas, pero en 6 casos (37,5\%) el diagnóstico fue posnatal $(29,29,32,35)$. La edad gestacional al momento del diagnóstico del hídrops está entre 23 y $35(27,33)$ semanas con un promedio de 28,9 semanas.

La anemia fetal severa se diagnosticó en tres casos mediante doppler de arteria cerebral media, requirieron transfusión intrauterina $(30,33,34)$ con una muerte intrauterina (33) y dos evolucionaron satisfactoriamente $(30,34)$.

\section{Tratamiento}

En cinco casos se realizó tratamiento durante el embarazo con penicilina, $(27,28,30,31,33)$. En 3 casos se administró prenatalmente penicilina benzatínica 2,400,000 UI IM semanal por tres dosis, $(27,28,33)$, en un caso se usó un esquema de penicilina cristalina 4 millones IV cada 6 horas por 14 días (30), y en un caso se administró inicialmente penicilina benzatí- nica y luego penicilina cristalina 4 millones IV cada 6 horas por 14 días (28).

En 11 casos no se administró tratamiento durante el embarazo $(26,28,29,32,34,35)$, en 6 casos porque el diagnóstico se realizó de manera posnatal $(26,29,32,35)$, y en 5 casos $(26,27,32,34)$ por el tiempo corto entre el diagnóstico y la necesidad de terminación del embarazo de emergencia.

La edad gestacional al momento del nacimiento fue entre 23 y 37 semanas con promedio de 30.9 semanas $(27,33)$. El peso de los nacidos fue de entre 720 y 3,540 gramos, con un promedio de $1,778 \mathrm{gr}$ (27-32). En cuatro casos la vía del parto fue vaginal $(26,30,32,33)$, y en 11 fue cesárea $(26,27,29,31,34,35)$. Un caso (28) no reporta la vía del parto. La mortalidad perinatal fue de seis casos $(37,5 \%)$, en tres casos se presentó muerte intrauterina $(26,32,33)$, y en otros tres se presentó muerte neonatal temprana $(26,29,35)$. En 10 casos el recién nacido sobrevivió $(26,27,28,30,31,32)$.

\section{CONCLUSIONES}

La sífilis congénita que cursa hídrops fetalis tiene una mortalidad cercana al 30\%, aun así, hay una buena respuesta con el tratamiento de primera elección que es la penicilina. El retraso en acudir al control 
prenatal y la tardanza del diagnóstico y tratamiento de la sífilis gestacional son causas importantes de la persistencia sífilis congénita. Se requieren estudios aleatorizados para determinar el mejor tratamiento del feto con sífilis congenia en los 30 días previos al parto y del feto con compromiso sistémico durante la segunda mitad de la gestación.

\section{REFERENCIAS}

1. WHO Guideline on Syphilis Screening and Treatment for Pregnant Women. Geneva: World Health Organization; 2017.Disponible en: https:/www.who.int/medical_devices/diagnostics/selection_in-vitro/selection_in-vitro-meetings/00007_02_WHO_Syphilis_SandT_Pregnant.pdf

2. Workowski KA, Bolan GA. Sexually transmitted diseases treatment guidelines, 2015 [errata publicada aparece en MMWR Recomm Rep. 2015;64(3):924. MMWR Recomm Rep. 2015;64(3):1-137. https:// doi.org/10.1056/NEJMra1901593.

3. Chakraborty R, Luck S. Syphilis is on the increase: the implications for child health. Arch Dis Child. 2008;93(2):1059. https://doi.org/10.1136/adc.2006.103515.

4. Adhikari EH. Syphilis in Pregnancy. Obstet Gynecol. 2020;135(5):1121-1135. https://doi.org/10.1097/ AOG.0000000000003788

5. Evans HE, Frenkel LD. Congenital syphilis. Clin Perinatol;21(1):149-62. https://doi.org/10.1016/ S0095-5108(18)30367-1.

6. Taylor M, Newman L, Ishikawa N, Laverty M, Hayashi C, Ghidinelli M, et al. Elimination of mother-tochild transmission of HIV and Syphilis (EMTCT): Process, progress, and program integration. PLoS Med. 2017;14(6):e1002329. https://doi.org/10.1371/ journal.pmed.1002329.

7. National Center for HIV/AIDS, Viral Hepatitis, STD, and TB Prevention (U.S.). Division of STD Prevention. Sexually transmitted disease surveillance 2018. National Center for HIV/AIDS, Viral Hepatitis, STD, and TB Prevention; 2019. https://doi.org/10.15620/cdc.79370.

8. Instituto Nacional de Salud. Boletín epidemiológico semanal 30 de 2020. Boletín Epidemiológico Semanal; 2020:1-25. https://doi. org/10.33610/23576189.2020.30.
9. Kidd S, Bowen VB, Torrone EA, Bolan G. Use of National Syphilis Surveillance Data to Develop a Congenital Syphilis Prevention Cascade and Estimate the Number of Potential Congenital Syphilis Cases Averted. Sex Transm Dis. 2018;45(9S Suppl 1):S23-S28. https://doi.org/10.1097/ OLQ.0000000000000838.

10. Slutsker JS, Hennessy RR, Schillinger JA. Factors Contributing to Congenital Syphilis Cases - New York City, 2010-2016. MMWR Morb Mortal Wkly Rep. 2018;67(39):1088-1093. https://doi.org/10.15585/ mmwr.mm6739a3.

11. Leichliter JS, Heyer K, Peterman TA, Habel MA, Brookmeyer KA, Arnold Pang SS, et al. US Public Sexually Transmitted Disease Clinical Services in an Era of Declining Public Health Funding: 2013-14. Sex Transm Dis. 2017;44(8):505-509. https://doi. org/10.1097/OLQ.0000000000000629.

12. Trivedi S, Williams C, Torrone E, Kidd S. National Trends and Reported Risk Factors Among Pregnant Women With Syphilis in the United States, 20122016. Obstet Gynecol. 2019;133(1):27-32. https:// doi.org/10.1097/AOG.0000000000003000

13. Ministerio de Salud y Protección Social . Guía de práctica clínica (GPC) basada en la evidencia para la atención integral de la sífilis gestacional y congénita. 2014. Disponible en: https:/www.minsalud.gov.co/ sites/rid/Lists/BibliotecaDigital/RIDE/INEC/IETS/ gpc\%20\%E2\%80\%93guia-completa-sifilis.pdf

14. Rac MWF, Bryant SN, McIntire DD, Cantey JB, Twickler DM, Wendel GD, et al. Progression of ultrasound findings of fetal syphilis after maternal treatment. Am J Obstet Gynecol. 2014;211(4):426.e1-426.e6. https:// doi.org/10.1016/j.ajog.2014.05.049.

15. Nathan L, Twickler DM, Peters MT, Sánchez PJ, Wendel GD. Fetal syphilis: Correlation of sonographic findings and rabbit infectivity testing of amniotic fluid. Obstet Gynecol Surv. 1993;48(11):730-731. https:// doi.org/10.1097/00006254-199311000-00008

16. Hollier LM, Harstad TW, Sanchez PJ, Twickler DM, Wendel GD. Fetal syphilis: Clinical and laboratory characteristics. Obstet Gynecol. 2001;97(6):947-953. https://doi.org/10.1016/S0029-7844(01)01367-9 
17. WHO Guidelines for the Treatment of Treponema pallidum (Syphilis). Geneva: World Health Organization; 2016.

18. Walker GJ, Walker D, Molano Franco D, GrilloArdila CF. Antibiotic treatment for newborns with congenital syphilis. Cochrane Database Syst Rev. 2019 Feb 15;2(2):CD012071. https://doi. org/10.1002/14651858.CD012071.pub2

19. New Zealand Sexual Health Society (NZSHS) Incorporated. Syphilis in PregnancyAntenatal Management Guidelines for maternal and congenital syphilis. 2020. Disponible en https://www.nzshs.org/docman/ guidelines/management-of-sexual-health-conditions/ syphilis/syphilis-in-pregnancy/397-syphilis-in-pregnancy-v1-sep-2020/file

20. Saloojee H, Velaphi S, Goga Y, Afadapa N, Steen R, Lincetto $O$. The prevention and management of congenital syphilis: an overview and recommendations. Bull World Health Organ. 2004 Jun;82(6):424-30.

21. Rac MW, Revell PA, Eppes CS. Syphilis during pregnancy: a preventable threat to maternal-fetal health. Am J Obstet Gynecol. 2017 Apr;216(4):352-363. https://doi.org/10.1016/j.ajog.2016.11.1052

22. Barron SD, Pass RF. Infectious causes of hydrops fetalis. Semin Perinatol. 1995 Dec;19(6):493-501. https://doi.org/10.1016/S0146-0005(05)80056-4

23. Insunza A, González R, Salvo A, Gómez R, Miland M, Rojas I, et al. Hídrops fetal no inmunológico secundario a sífilis fetal: diagnóstico y tratamiento antenatal Rev. chil. obstet. ginecol; 61(5): 317-22, 1996. ID: lil-194469

24. ElTabbakh GH, Elejalde BR, Broekhuizen FF. Primary syphilis and nonimmune fetal hydrops in a penicillin-allergic woman. A case report. J Reprod Med. 1994;39(5):412-414. PMID: 8064710

25. Hallak M, Peipert JF, Ludomirsky A, Byers J. Nonimmune hydrops fetalis and fetal congenital syphilis. A case report.J Reprod Med. 1992;37(2):173-176. PMID: 1538364

26. Berkowitz K, Baxi L, Fox HE. False-negative syphilis screening: the prozone phenomenon, nonimmune hydrops, and diagnosis of syphilis during pregnancy. Am J Obstet Gynecol. 1990;163(3):975-977. https:// doi.org/10.1016/0002-9378(90)91107-N
27. Barton JR, Thorpe EM Jr, Shaver DC, Hager WD, Sibai BM. Nonimmune hydrops fetalis associated with maternal infection with syphilis. Am J Obstet Gynecol. 1992;167(1):56-58. https://doi.org/10.1016/S00029378(11)91625-1

28. Galan HL, Yandell PM, Knight AB. Intravenous penicillin for antenatal syphilotherapy. Infect Dis Obstet Gynecol. 1993;1(1):7-11. https://doi.org/10.1155/ S1064744993000031

29. Levine Z, Sherer DM, Jacobs A, Rotenberg O. Nonimmune hydrops fetalis due to congenital syphilis associated with negative intrapartum maternal serology screening. Am J Perinatol. 1998;15(4):233-236. https:// doi.org/10.1055/s-2007-993933

30. Chen I, Chandra S, Singh A, Kumar M, Jain V, Turnell $R$. Successful outcome with intrauterine transfusion in non-immune hydrops fetalis secondary to congenital syphilis. J Obstet Gynaecol Can. 2010;32(9):861-865. https://doi.org/10.1016/S1701-2163(16)34658-8

31. Araujo Júnior E, Martins Santana EF, Rolo LC, Nardozza LM, Moron AF. Prenatal diagnosis of congenital syphilis using two- and three-dimensional ultrasonography: case report. Case Rep Infect Dis. 2012;2012:478436. https://doi.org/10.1155/2012/478436

32. Macé G, Castaigne V, Trabbia A, et al. Fetal anemia as a signal of congenital syphilis. J Matern Fetal Neonatal Med. 2014;27(13):1375-1377. https://doi.org/10.31 09/14767058.2013.853288

33. Fuchs F, Michaux K, Rousseau C, Ovetchkine P, Audibert F. Syphilis Infection: An Uncommon Etiology of Infectious Nonimmune Fetal Hydrops with Anemia. Fetal Diagn Ther. 2016;39(1):74-77. https://doi. org/10.1159/000364804

34. Duby J, Bitnun A, Shah V, Shannon P, Shinar S, Whyte H. Non-immune Hydrops Fetalis and Hepatic Dysfunction in a Preterm Infant With Congenital Syphilis. Front Pediatr. 2019;7:508. Published 2019 Dec 11. https://doi.org/10.3389/fped.2019.00508

35. Ramis Fernández SM, Alsina-Casanova M, HerranzBarbero A, Aldecoa-Bilbao V, Borràs-Novell C, SalviaRoges D. Hydrops fetalis caused by congenital syphilis: An ancient disease?. Int J STD AIDS. 2019;30(14):14361439. https://doi.org/10.1177/0956462419876487 


\section{CONTRIBUCIÓN DE LOS} AUTORES

Andrés Mauricio Camacho-Montaño: elaboración del documento desde su concepción y diseño hasta la adquisición de la información, revisión del contenido intelectual y aprobación de la versión enviada a proceso editorial.
Reinaldo Niño-Alba: elaboración del documento desde su concepción y diseño hasta la adquisición de la información, revisión del contenido intelectual y aprobación de la versión enviada a proceso editorial. Edgar Páez-Castellanos: elaboración del documento desde su concepción y diseño hasta la adquisición de la información, revisión del contenido intelectual y aprobación de la versión enviada a proceso editorial. 